\title{
Guest Editorial Foreword to the Special Issue on Virtual, Augmented, and Mixed Reality Applications
}

This special issue of the JIS (SBC Journal on 3D Interactive Systems) is for the third year acknowledging the best papers of the Symposium on Virtual and Augmented Reality (SVR). The SVR is the most important Brazilian conference about Virtual, Augmented and Mixed Reality, which is being conducted by acadeThis special issue of the SBC Journal on 3D Interactive Systems is dedicated to Virtual, Augmented, and Mixed Reality Applications. Our goal is to present systems papers focusing on applications and on how they are being used (or how they are intended to be used) to solve real problems.

We received seven manuscripts and after a peer review phase, were able to select five papers. The selected papers for this issue cover very different areas, ranging from industry to digital art, through medicine, spacecraft simulation and terrain generation.

The first paper, by Thalen and van der Voort, from the University of Twente, Nederlands, presents an industrial VR application for the early stages of product development stage. They claim that VR can facilitate communication between designers and product end-users to improve the quality of feedback that user provide to designers. Moving from industry to medicine, the second paper in this special issue, by Torres et al., from the State University of São Paulo (USP), introduces a serious game to simulate breast biopsy exams using VR. The third paper, by Guimarães, Dias, and Gnecco, describes the experience of transporting a hyper-realistic painting by the Brazilian plastic artist Sônia Menna Barreto into the digital world and bringing her work to life by means of VR technologies. The fourth paper, by Dembogurski et al., from the Federal University of Juiz de Fora (UFJF) and the Fluminense Federal University (UFF) introduces an application to generate virtual terrains interactively using AR markers and also supports user navigation in the generated virtual environment. From land to space, the last paper of this special issue, by Cerqueira, dos Santos, and Ambrosio, from the Brazilian National Institute for Space Research (INPE), describes a goal-driven user interface for spacecraft distributed simulations using a service oriented paradigm to support different space missions.
Finally, we would like to express our thanks to the editorin-chief, Luciana Nedel, for inviting us to be guest editors of this special issue. We would like also to thank the reviewers for their help and insightful comments. Last but not the least, we are grateful to the authors for their contributions and their willingness to participate in the laborious process of preparing this issue.

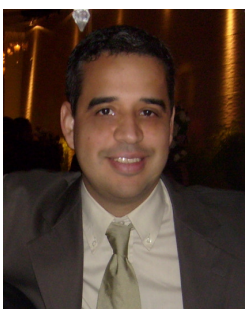

Alberto Raposo is Assistant Professor at the Dept. of Informatics / PUC-Rio and project coordinator at Tecgraf/PUCRio. DSc in Electrical/Computer Engineering at the State University of Campinas, Brazil. Current interests: Virtual Reality, 3D interaction, groupware, $\mathrm{HCl}$, and computer graphics, with more than 120 publications in these areas. Projects supported by: Petrobras, CNPq, FINEP, FAPERJ and RNP. Distinguished young scholar, PUC-Rio and NVIDIA Academic Partner.

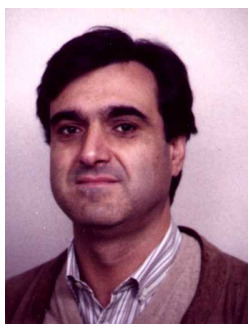

Joaquim Jorge is Professor at Instituto Superior Técnico (IST/UTL), the School of Engineering of the Technical University of Lisboa, Portugal, where he teaches User Interfaces and Computer Graphics. He received the PhD and MSc degrees in Computer Science from Rensselaer Polytechnic Institute, Troy, NY, in 1995 and a BsEE from IST/UTL in 1984. He is author or co-author of more than 221 papers published in peer-reviewed international conferences, journals and books. A Senior Member of IEEE and ACM, he was elected Fellow of the Eurographics Association in 2010.

Alberto B. Raposo - PUC-Rio abraposo@inf.puc-rio.br

Joaquim A. P. Jorge - IST/UTL jaj@inesc.pt 